\title{
Traditional and Modern Medicine Harmonizing the Two Approaches in the Treatment of Neurodegeneration (Alzheimer's Disease - AD)
}

\author{
Bowirrat Abdalla, Mustafa Yassin, Menachem Abir, \\ Bishara Bisharat and Zaher Armaly
}

Additional information is available at the end of the chapter

http://dx.doi.org/10.5772/48558

\section{Introduction}

Neurodegenerative disorders, Primarily, are multifactorial diseases characterized by chronic and progressive loss of neurons in discrete areas of the brain, causing debilitating symptoms and globally decreasing cognitive function such as dementia, loss of memory, loss of sensory or motor capability, decreased overall quality of life and well-being, disability, and eventually, premature death. For most neurodegenerative diseases, there is little or no treatment; at best, treatments are symptomatic in nature and do not prevent or slow the progression of disease. Clearly, an understanding of pathological progression can help to identify points of intervention and lead to promising therapeutic approaches. A fundamental approach for reducing the burden of neurodegenerative diseases is thus to slow or halt progression, and ultimately, to prevent the onset of the disease process. Strategies for neurorescue, neurorepair, neuroprotection or treatment are being actively pursued by the basic, translational, and clinical research communities. As our population ages, the already enormous impact of neurodegeneration on society will become even larger without better prevention and treatment.

"Dementia" is an umbrella term describing a variety of diseases and conditions that develop when nerve cells in the brain die or no longer function normally. The death or malfunction of these nerve cells, called neurons, causes changes in one's memory, behavior and Ability to think clearly. In Alzheimer's disease (AD), these brain changes eventually impair an individual's Ability to carry out such basic bodily functions as walking and swallowing. As aged population dramatically increases in these decades, efforts should be made on the intervention for curing age-associated neurodegenerative diseases such as AD. 
$\mathrm{AD}$ is considered to be the most widespread variety of dementia $(57 \%-65 \%)$ or a condition typified by continuous decline of mental aptitudes $(1,2)$.

$\mathrm{AD}$ affects about 5.4 million people in the United States alone, and that number is projected to reach 12-16 million by the year 2050 (3). Economically, AD is a major public health problem. In the United States in 2011, the cost of health care, long-term care, and hospice services for people aged 65 years and older with $\mathrm{AD}$ and other dementias was expected to be $\$ 183$ billion, and this figure does not include the contributions of unpaid caregivers (3).

Currently, an autopsy or brain biopsy is the only way to make a definitive diagnosis of AD. In clinical practice, the diagnosis is usually made on the basis of the history and findings on Mental Status Examination.

Symptomatic therapies are the only treatments available for AD. The standard medical treatments include cholinesterase inhibitors and a partial $N$-methyl-D-aspartate (NMDA) antagonist. Psychotropic medications are often used to treat secondary symptoms of AD, such as depression, agitation, and sleep disorders.

\section{Historical background}

In 1901, a German psychiatrist named Alois Alzheimer observed a patient at the Frankfurt Asylum named Mrs. Auguste D. This 51-year-old woman suffered from a loss of short-term memory, among other behavioral symptoms that puzzled Dr. Alzheimer. Five years later, in April 1906, the patient died, and Dr. Alzheimer sent her brain and her medical records to Munich, where he was working in the lab of Dr. Emil Kraeplin. By staining sections of her brain in the laboratory, he was able to identify amyloid plaques and neurofibrillary tangles (4).

A speech given by Dr. Alzheimer on November 3, 1906, was the first time the pathology and the clinical symptoms of the disorder, which at the time was termed presenile dementia, were presented together. Alzheimer published his findings in 1907 (5).

In the past 15-20 years, dramatic progress has been made in understanding the neurogenetics and pathophysiology of AD. Four different genes have been definitively associated with $\mathrm{AD}$, and others that have a probable role have been identified. The mechanisms by which altered amyloid and tau protein metabolism, inflammation, oxidative stress, and hormonal changes may produce neuronal degeneration in $\mathrm{AD}$ are being elucidated, and rational pharmacologic interventions based on these discoveries are being developed.

Rapid progress towards understanding the molecular underpinnings of neurodegenerative disorders such as $\mathrm{AD}$ is revolutionizing drug discovery for these conditions. Furthermore, the development of models for these disorders is accelerating efforts to translate insights related to neurodegenerative mechanisms into disease-modifying therapies.

$\mathrm{AD}$ or Alzheimer's-type dementia is a progressive degeneration of brain tissue that primarily strikes people over age 65. It is the most common cause of dementia and is marked by a devastating mental decline. Intellectual functions such as memory, 
comprehension, and speech deteriorate. Attention tends to stray, simple calculations become impossible, and ordinary daily activities grow increasingly difficult, accompanied by bewilderment and frustration.

$\mathrm{AD}$ characterized clinically by progressive memory deficits, impaired cognitive function, and altered and inappropriate behavior. AD places a considerable and increasing burden on patients, caregivers, and society. Aging represents the most important risk factor and dementia has become one of the major challenges in our societies due to the universal phenomenon of population aging in the world. Brain regions involved in learning and memory processes, including the temporal and frontal lobes as well as the hippocampus, are reduced in size in $\mathrm{AD}$ patients as the result of degeneration of synapses and death of neurons. AD is considered as a protein aggregation disorder, based on two key neuropathological hallmarks, namely the hyperphosphorylation of the tau protein resulting in the formation of neurofibrillary tangles (NFTs), and the increased formation and aggregation of amyloid-beta peptide $(\mathrm{A} \beta)$ derived from amyloid precursor protein (APP) (6).

Although the exact underlying cause initiating the onset of AD is still unclear, an imbalance in oxidative and nitrosative stress, intimately linked to mitochondrial dysfunction, characterizes already early stages of AD pathology.

\section{Etiology of Alzheimer's Disease (AD)}

The cause of $\mathrm{AD}$ is not entirely known, but is thought to include both genetic and environmental factors (Multifactorial). A diagnosis of AD is made when certain symptoms are present, and by making sure other causes of dementia are not present (DSM-IV criteria).

The only way to know for certain that someone has AD is to examine a sample of their brain tissue after death. The following changes are more common in the brain tissue of people with AD:

1. "Neurofibrillary tangles" (twisted fragments of protein within nerve cells that clog up the cell)

2. "Neuritic plaques" (Abnormal clusters of dead and dying nerve cells, other brain cells, and protein)

3. "Senile plaques" (areas where products of dying nerve cells have accumulated around protein).

When nerve cells (neurons) are destroyed, there is a decrease in the neurotransmitters. As a result, areas of the brain that normally work together become disconnected.

Healthy neurons have an internal support structure partly made up of structures called microtubules. These microtubules act like tracks, guiding nutrients and molecules from the body of the cell down to the ends of the axon and back. A special kind of protein, tau, binds to the microtubules and stabilizes them.

In $\mathrm{AD}$, tau is changed chemically. It begins to pair with other threads of tau, which become tangled together. When this happens, the microtubules disintegrate, collapsing the neuron's 
transport system (see the image below). The formation of these neurofibrillary tangles (NFTs) may result first in malfunctions in communication between neurons and later in the death of the cells.

In addition to NFTs, the anatomic pathology of AD includes senile plaques (SPs; also known as beta-amyloid plaques) at the microscopic level and cerebrocortical atrophy at the macroscopic level (see the image below). The hippocampus and medial temporal lobe are the initial sites of tangle deposition and atrophy (7). This can be seen on brain magnetic resonance imaging early in $\mathrm{AD}$ and helps support a clinical diagnosis.

SPs and NFTs were described by Alois Alzheimer in his original report on the disorder in 1907 (5). They are now universally accepted as the pathological hallmark of the disease.

A continuum exists between the pathophysiology of normal aging and that of AD (8). Pathologic hallmarks of AD have been identified; however, these features also occur in the brains of cognitively intact persons. For example, in a study in which neuropathologists were blinded to clinical data, they identified $76 \%$ of brains of cognitively intact elderly patients as demonstrating $\mathrm{AD}(9)$.

$\mathrm{AD}$ affects the 3 processes that keep neurons healthy: communication, metabolism, and repair. Certain nerve cells in the brain stop working, lose connections with other nerve cells, and finally die. The destruction and death of these nerve cells causes the memory failure, personality changes, problems in carrying out daily activities, and other features of the disease.

The accumulation of SPs primarily precedes the clinical onset of AD. NFTs, loss of neurons, and loss of synapses accompany the progression of cognitive decline (8).

Considerable attention has been devoted to elucidating the composition of SPs and NFTs to find clues about the molecular pathogenesis and biochemistry of AD. The main constituent of NFTs is the microtubule-associated protein tau (see Anatomy). In AD, hyperphosphorylated tau accumulates in the perikarya of large and medium pyramidal neurons. Somewhat surprisingly, mutations of the tau gene result not in AD but in some familial cases of frontotemporal dementia.

Since the time of Alois Alzheimer, SPs have been known to include a starchlike (or amyloid) substance, usually in the center of these lesions. The amyloid substance is surrounded by a halo or layer of degenerating (dystrophic) neurites and reactive glia (both astrocytes and microglia).

One of the most important advances in recent decades has been the chemical characterization of this amyloid protein, the sequencing of its amino acid chain, and the cloning of the gene encoding its precursor protein (on chromosome 21). These advances have provided a wealth of information about the mechanisms underlying amyloid deposition in the brain, including information about the familial forms of AD.

Although the amyloid cascade hypothesis has gathered the most research financing, other interesting hypotheses have been proposed. Among these are the mitochondrial cascade hypotheses (10). 
In addition to NFTs and SPs, many other lesions of AD have been recognized since Alzheimer's original papers were published. These include the granulovacuolar degeneration of Shimkowicz; the neuropil threads of Braak et al (11); and neuronal loss and synaptic degeneration, which are thought to ultimately mediate the cognitive and behavioral manifestations of the disorder.

\subsection{Neurofibriliary tangles and senile plaques}

Plaques are dense, mostly insoluble deposits of protein and cellular material outside and around the neurons. Plaques are made of beta-amyloid $(\mathrm{A} \beta)$, a protein fragment snipped from a larger protein called amyloid precursor protein (APP). These fragments clump together and are mixed with other molecules, neurons, and non-nerve cells.
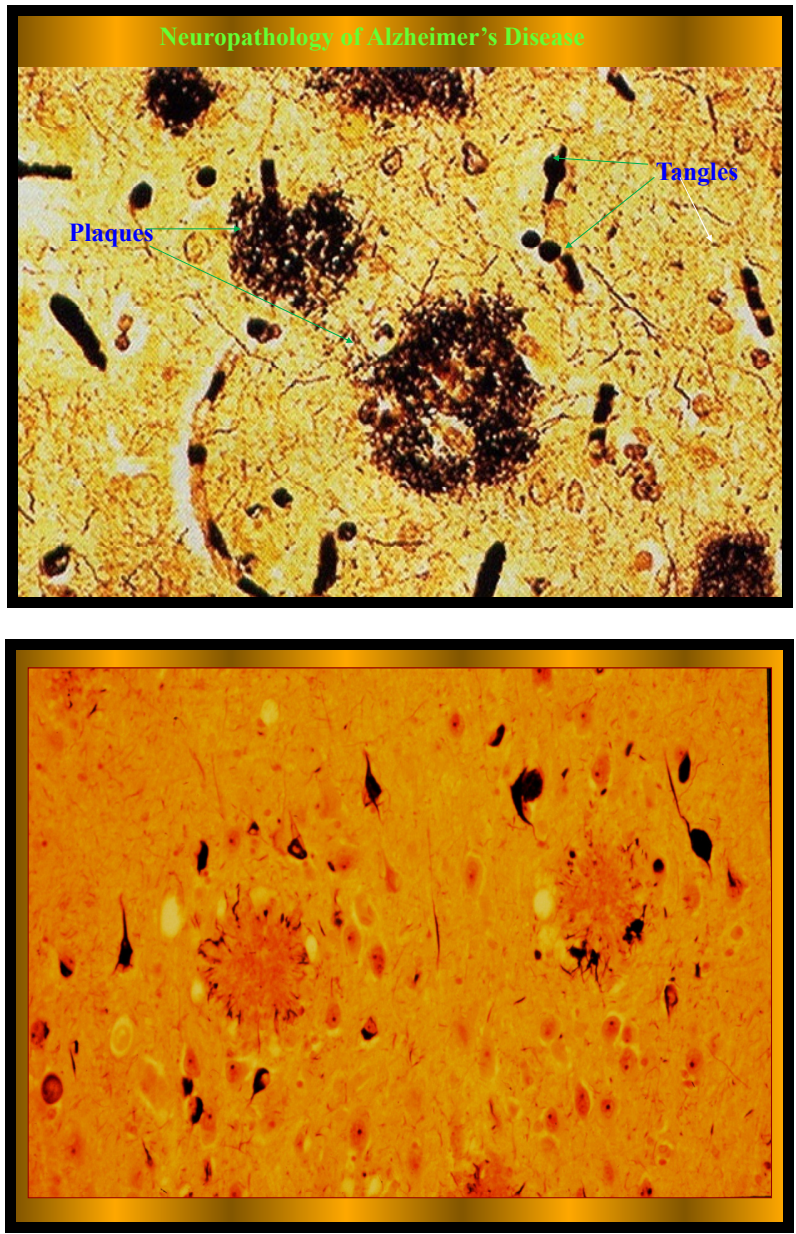

Figure 1. Amyloid plaques. 


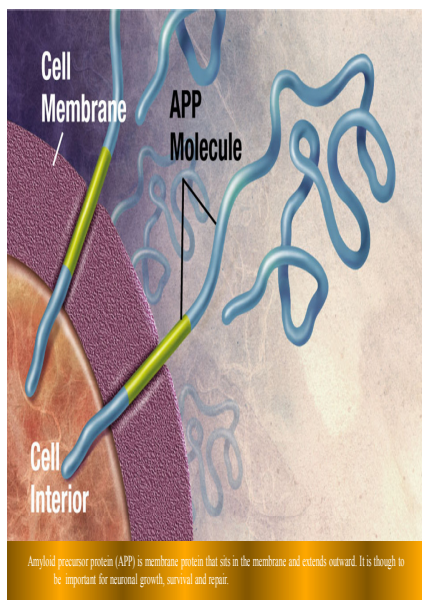

1

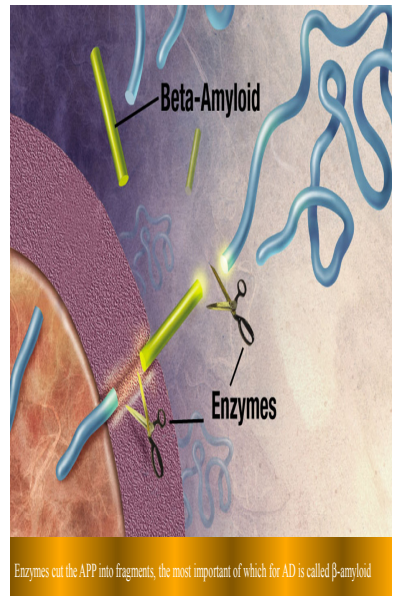

2

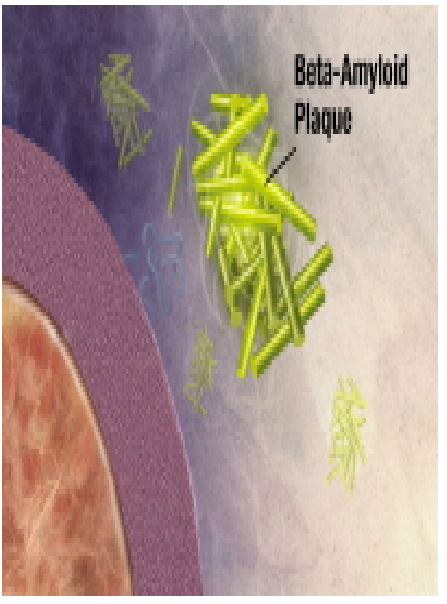

3

1. APP sticks through the neuron membrane.

2. Enzymes cut the APP into fragments of protein, including beta-amyloid.

3. Beta-amyloid fragments come together in clumps to form plaques.

Figure 2. Amyloid precursor protein (APP) is the precursor to amyloid plaque.

In $\mathrm{AD}$, plaques develop in the hippocampus, a structure deep in the brain that helps to encode memories, and in other areas of the cerebral cortex that are used in thinking and making decisions. Plaques may begin to develop as early as the fifth decade of life (12). Whether $\mathrm{A} \beta$ plaques themselves cause $\mathrm{AD}$ or whether they are a by-product of the $\mathrm{AD}$ process is still unknown. It is known that changes in APP structure can cause a rare, inherited form of AD.

Tangles are insoluble twisted fibers that build up inside the nerve cell. Although many older people develop some plaques and tangles, the brains of people with $\mathrm{AD}$ have them to a greater extent, especially in certain regions of the brain that are important in memory. There are likely to be significant age-related differences in the extent to which the presence of plaques and tangles are indicative of the presence of dementia.

NFTs are initially and most densely distributed in the medial aspect and in the pole of the temporal lobe; they affect the entorhinal cortex and the hippocampus most severely (however, Braak et al found that in sporadic AD, tauopathy may appear first in the lower brainstem rather than in the transentorhinal region (12). As AD progresses, NFTs accumulate in many other cortical regions, beginning in high-order association regions and less frequently in the primary motor and sensory regions.

SPs also accumulate primarily in association cortices and in the hippocampus. Plaques and tangles have relatively discrete and stereotypical patterns of laminar distribution in the cerebral cortex, which indicate predominant involvement of corticocortical connections. 
Although NFTs and SPs are characteristic of AD, they are not pathognomonic. NFTs are found in several other neurodegenerative disorders, including progressive supranuclear palsy and dementia pugilistica (chronic traumatic encephalopathy). SPs may occur in normal aging.

Therefore, the mere presence of these lesions is not sufficient to support the diagnosis of AD. These lesions must be present in sufficient numbers and in a characteristic topographic distribution to fulfill the current histopathologic criteria for AD. There is consensus that the presence of even low numbers of NFTs in the cerebral neocortex with concomitant SPs is characteristic of AD.

Some authorities believed that NFTs, when present in low densities and essentially confined to the hippocampus, were part of normal aging. However, the histologic stages for AD that Braak et al formulated include an early stage in which NFTs are present at a low density in the entorhinal and perirhinal (ie, transentorhinal) cortices (12). Therefore, even small numbers of NFTs in these areas of the medial temporal lobe may be abnormal.

\section{Amyloid hypothesis versus tau hypothesis}

A central but controversial issue in the pathogenesis of $\mathrm{AD}$ is the relationship between amyloid deposition and NFT formation. Evidence shows that abnormal amyloid metabolism plays a key pathogenic role. At high concentrations, the fibrillar form of $A \beta$ has been shown to be neurotoxic to cultured neurons.

Cultured cortical and hippocampal neurons treated with $\mathrm{A} \beta$ protein exhibit changes characteristic of apoptosis (self-regulated cell destruction), including nuclear chromatin condensation, plasma membrane blebbing, and internucleosomal DNA fragmentation. The fibrillar form of $\mathrm{A} \beta$ has also been shown to alter the phosphorylation state of tau protein.

The identification of several point mutations within the APP gene in some patients with early-onset familial $\mathrm{AD}$ and the development of transgenic mice exhibiting cognitive changes and SPs also incriminate A $\beta$ in AD. The apolipoprotein E (APOE) E4 allele, which has been linked with significantly increased risk for developing $A D$, may promote inability to suppress production of amyloid, increased production of amyloid, or impaired clearance of amyloid with collection outside of the neuron.

Autopsies have shown that patients with 1 or 2 copies of the APOE E4 allele tend to have more amyloid. Additional evidence comes from recent experimental data supporting the role of presenilins in $A \beta$ metabolism, as well as findings of abnormal production of $A \beta$ protein in presenilin-mutation familial AD.

Although very popular, the amyloid hypothesis is not uniformly accepted. On post-mortem analysis, amyloid plaques may be undetectable in the brains of patients who had severe AD but may be present in the brains of elderly patients who did not have dementia (13).

Dementia severity correlates better with the number of neocortical NFTs than with SPs. The tau protein stabilizes neuronal microtubules. Destabilization of the microtubular system is 
speculated to disrupt the Golgi apparatus, in turn inducing abnormal protein processing and increasing production of $\mathrm{A} \beta$. In addition, this destabilization may decrease axoplasmic flow, generating dystrophic neurites and contributing to synaptic loss.

\subsection{Granulovacuolar degeneration and neuropil threats}

Granulovacuolar degeneration occurs almost exclusively in the hippocampus. Neuropil threads are an array of dystrophic neurites diffusely distributed in the cortical neuropil, more or less independently of plaques and tangles. This lesion suggests neuropil alterations beyond those merely due to NFTs and SPs and indicates an even more widespread insult to the cortical circuitry than that visualized by studying only plaques and tangles.

\subsection{Cholinergic neurotransmission and Alzheimer disease}

The cholinergic system is involved in memory function, and cholinergic deficiency has been implicated in the cognitive decline and behavioral changes of AD. Activity of the synthetic enzyme choline acetyltransferase (CAT) and the catabolic enzyme acetylcholinesterase are significantly reduced in the cerebral cortex, hippocampus, and amygdala in patients with AD.

The nucleus basalis of Meynert and diagonal band of Broca provide the main cholinergic input to the hippocampus, amygdala, and neocortex, which are lost in patients with AD. Loss of cortical CAT and decline in acetylcholine synthesis in biopsy specimens have been found to correlate with cognitive impairment and reaction-time performance. Because cholinergic dysfunction may contribute to the symptoms of patients with AD, enhancing cholinergic neurotransmission constitutes a rational basis for symptomatic treatment.

\subsection{Oxidative stress and damage}

Oxidative damage occurs in AD. Studies have demonstrated that an increase in oxidative damage selectively occurs within the brain regions involved in regulating cognitive performance (14).

Oxidative damage potentially serves as an early event that then initiates the development of cognitive disturbances and pathological features observed in AD. A decline in protein synthesis capabilities occurs in the same brain regions that exhibit increased levels of oxidative damage in patients with mild cognitive impairment (MCI) and AD. Protein synthesis may be one of the earliest cellular processes disrupted by oxidative damage in AD (15).

Oxidative stress is believed to be a critical factor in normal aging and in neurodegenerative diseases such as Parkinson disease, amyotrophic lateral sclerosis, and AD.

The apoptotic pattern of cellular death seen in oxidative stress is similar to that produced by $\mathrm{A} \beta$ peptide exposure, and $\mathrm{A} \beta$ neurotoxicity is attenuated by antioxidants such as vitamin $\mathrm{E}$. $\mathrm{A} \beta$ may induce toxicity by engaging several binding sites on the membrane surface. 
Several investigators now believe that converging environmental and genetic risk factors trigger a pathophysiologic cascade that, over decades, leads to Alzheimer pathology and dementia.

The following risk factors for Alzheimer-type dementia have been identified: Advancing age;Family history; APOE 4 genotype; Obesity; Insulin resistance; Vascular factors; Dyslipidemia; Hypertension; Inflammatory markers; Down syndrome and Traumatic brain injury (16-19).

In addition, epidemiologic studies have suggested some possible risk factors (eg, aluminum $(20,21)$, previous depression) and some protective factors (eg, education $(22,23)$, long-term use of nonsteroidal anti-inflammatory drugs (24).

\section{Genetics causes}

Although most cases of $\mathrm{AD}$ are sporadic (ie, not inherited), familial forms of $\mathrm{AD}$ do exist. Autosomal dominant AD, which accounts for less than $5 \%$ of cases, is almost exclusively early onset $\mathrm{AD}$; cases occur in at least 3 individuals in 2 or more generations, with 2 of the individuals being first-degree relatives (25).

Familial clustering represents approximately $15-25 \%$ of late-onset AD cases and most often involves late-onset AD. In familial clustering, at least 2 of the affected individuals are thirddegree relatives or closer (25).

Mutations in the following genes unequivocally cause early-onset autosomal dominant AD:

The amyloid precursor protein $(A P P)$ gene on chromosome 21

The presenilin-1 (PS1) gene on chromosome 14

The presenilin-2 (PS2) gene on chromosome 1

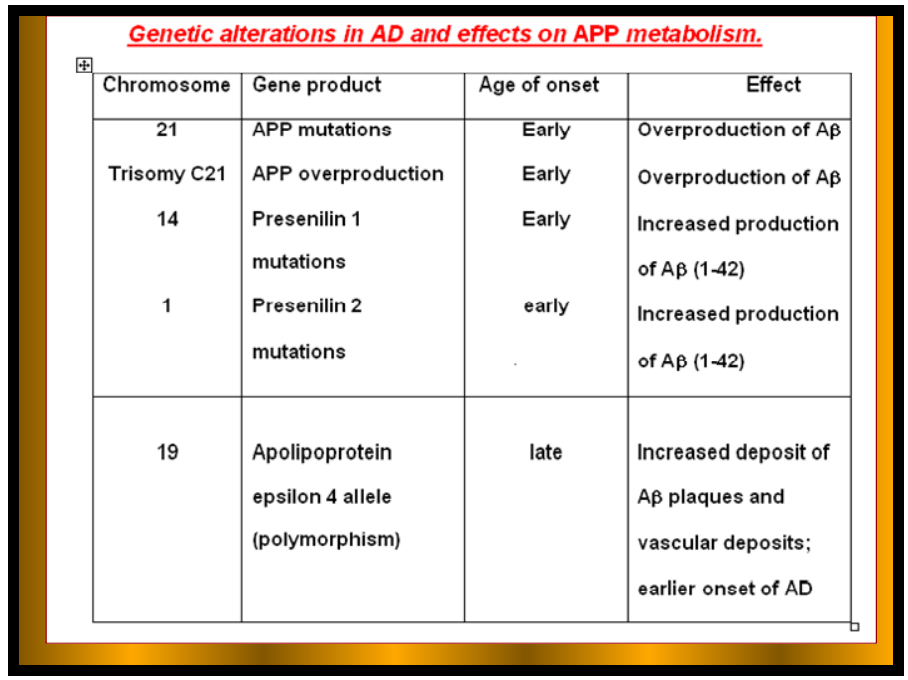


All 3 of these genes lead to a relative excess in the production of the stickier 42 -amino acid form of the $\mathrm{A} \beta$ peptide over the less sticky 40-amino-acid form.

This beta-pleated peptide is postulated to have neurotoxic properties and to lead to a cascade of events (as yet incompletely understood) that results in neuronal death, synapse loss, and the formation of NFTs and SPs, among other lesions. Nonetheless, the mutations that have been found to date account for less than half of all cases of early-onset AD.

Other than the apolipoprotein E epsilon 4 (APOE E4) genotype, no polymorphisms in other genes have been consistently found to be associated with late-onset AD. However, genomewide association studies have identified the following additional susceptibility loci (26).

\section{APP mutations}

The observation that patients with Down syndrome (trisomy 21) develop cognitive deterioration and typical pathological features of $\mathrm{AD}$ by middle age led to the discovery of the $A P P$ gene on chromosome 21. Simultaneously, a locus segregating with a minority of earlyonset familial AD kindreds was mapped to this chromosome, in the same region as the APP gene.

Subsequently, several missense mutations within the APP gene that resulted in amino acid substitutions in APP were identified in these familial AD kindreds. Such mutations appear to alter the previously described proteolytic processing of APP, generating amyloidogenic forms of $A \beta$.

Skin fibroblasts from individuals carrying $A P P$ mutations produce increased $A \beta$ 42/43. Increased plasma concentration of $A \beta 42 / 43$ is also seen in these patients, regardless of age, sex, or clinical status. Interestingly, some patients with sporadic AD may exhibit similar elevations of plasma $A \beta 42 / 43$.

\section{PS1 and PS2 mutations}

Approximately 50-70\% of early-onset autosomal-dominant AD cases appear to be associated with a locus (AD3) mapped by genetic linkage to the long arm of chromosome 14 (14q24.3). Numerous missense mutations have been identified on a strong candidate gene, called PS1.

At the same time, another autosomal dominant locus responsible for early-onset AD was localized to chromosome 1. Two mutations were identified on the candidate gene, designated PS2. The physiological role of presenilins and the pathogenic effects of their mutations are not yet well understood.

\subsection{APOE}

The gene encoding the cholesterol-carrying apolipoprotein $\mathrm{E}(A P O E)$ on chromosome 19 has been linked to increased risk for $\mathrm{AD}$, principally late-onset but also some early-onset cases. The gene is inherited as an autosomal codominant trait with 3 alleles. The APOE E2 allele, 
the least prevalent of the 3 common APOE alleles, is associated with the lowest risk of developing $\mathrm{AD}$ (27), with a lower rate of annual hippocampal atrophy and higher cerebrospinal fluid $A \beta$ and lower phosphotau, suggesting less AD pathology (28).

The E3 allele confers intermediate risk of developing AD, with less risk than the E4 allele. The E3 allele, which is more common than the E2 allele, may protect tau from hyperphosphorylation, and the E2 allele's effect on tau phosphorylation is complex.

APOE E4 gene "dose" is correlated with increased risk and earlier onset of AD (29). Persons with 2 copies of the APOE E4 allele (4/4 genotype) have a significantly greater risk of developing AD than persons with other APOE subtypes. Mean age at onset is significantly lower in the presence of 2 APOE E4 copies. A collaborative study has suggested that APOE E4 exerts its maximal effect before the age of 70 years.

Many APOE E4 carriers do not develop AD, and many patients with AD do not have this allele. Therefore, the presence of an APOE E4 allele does not secure the diagnosis of AD, but instead, the APOE E4 allele acts as a biological risk factor for the disease, especially in those younger than 70 years.

\section{Treatment of Alzheimer's disease - From medical chemistry to plants}

The worldwide population and especially the wesearn and U.S. population is getting older, and as it ages, $\mathrm{AD}$ is becoming an increasingly bigger concern. Within the next 50 years, the incidence of Alzheimer's is expected to quadruple, affecting one in 45 Americans.

Today, there is still no cure for Alzheimer's. People with the disease progressively lose memory and the ability to function as Alzheimer's advances.

Several different types of medications are used to treat the memory loss, behavior changes, sleep problems, and other symptoms of AD. These medications won't stop the disease, but they can slow down the symptoms for a few months or even years. All of these medications can have side effects, which can be even more pronounced in the elderly.

Early diagnosis and treatment allows AD patients to maintain the highest levels of cognitive and functional ability possible.

Today main pharmacological treatment of $\mathrm{AD}$ is Cholinesterase inhibitors (ChEIs), and mental exercises are used in an attempt to prevent or delay the deterioration of cognition in patients with AD. Here, we will try to shed lights on the important available pharmacological treatments and complementary therapies such as Herbal medicine utilized on the treatment of $\mathrm{AD}$ :

\subsection{Pharmacological treatment}

\section{A. Cholinesterase inhibition}

Numerous lines of evidence suggest that cholinergic systems that modulate information processing in the hippocampus and neocortex are impaired early in the course of AD. These 
observations have suggested that some of the clinical manifestations of AD are due to loss of cholinergic innervation to the cerebral cortex.

Centrally acting ChEIs prevent the breakdown of acetylcholine. Four such agents have been approved by the FDA for the treatment of AD, as follows:

Tacrine

Donepezil (Aricept, Aricept ODT)

Rivastigmine (Exelon, Exelon Patch)

Galantamine (Razadyne, Razadyne ER)

Of note, tacrine has potential hepatotoxicity and hence requires frequent blood monitoring. Since the other ChEIs have become available, tacrine has rarely been prescribed.

All ChEIs have shown modest benefit on measures of cognitive function and activities of daily living. Patients on ChEIs have shown slower declines on cognitive and functional measures than patients on placebo. However, ChEIs do not address the underlying cause of the degeneration of cholinergic neurons, which continues during the disease. The ChEIs may also alleviate the noncognitive manifestations of $\mathrm{AD}$, such as agitation, wandering, and socially inappropriate behavior (30).

Although the usefulness of ChEIs was originally expected to be limited to the early and intermediate stages of $\mathrm{AD}$ (because the cholinergic deficit becomes more severe later in disease and because fewer intact cholinergic synapses are present), they are also helpful in advanced disease (31). ChEIs are also helpful in patients with AD with concomitant infarcts and in patients with dementia with Lewy bodies. Frequently, AD and dementia with Lewy bodies occur in the same patient; this is sometimes called the Lewy body variant of AD.

The ChEIs share a common profile of adverse effects, the most frequent of which are nausea, vomiting, diarrhea, and dizziness. These are typically dose related and can be mitigated with slow up-titration to the desired maintenance dose. In addition, gastrointestinal side effects may be reduced by using the transdermal patch rather than the oral form of the drug. As antimuscarinic drugs are used for the treatment of incontinence, logically, ChEIs might exacerbate incontinence. One brief report has supported this hypothesis (32).

ChEIs prescribed to treat dementia can provoke symptomatic bradycardia and syncope and precipitate fall-related injuries, including hip fracture. In a study of older adults with dementia who were taking cholinesterase inhibitors, hospital visits for syncope were found to be more frequent in patients receiving ChEIs than in control patients (31.5 vs 18.6 events per 1000 person-years (33). Other syncope-related events, including hospital visits for bradycardia, permanent pacemaker insertion, and hip fracture, were also found to be more common in patients receiving cholinesterase inhibitors. ChEI use in older adults with dementia is associated with increased risk of syncope-related events; these risks must be weighed against the benefits of taking ChEIs (33).

Anecdotal reports exist of acute cognitive and behavioral decline associated with the abrupt termination of ChEIs. In several of these cases, restarting the ChEI did not lead to substantial 
improvement. These reports have implications concerning the best practice when switching a patient from one ChEI to another in this class. Reasons for switching might include undesirable side effects or an apparent lack of efficacy. Nonetheless, no published data are available to help clinicians know when it would be helpful to switch to another ChEI.

The common practice of tapering a patient off one CNS-active medication before starting a new one should not be followed when changing ChEIs. For example, a patient who was taking $10 \mathrm{mg}$ of donepezil should be started the next day on galantamine at a dose of at least $8 \mathrm{mg} /$ day and possibly $16 \mathrm{mg} /$ day. No current evidence supports the use of more than 1 ChEI at a time. Centrally acting anticholinergic medications should be avoided.

It is not uncommon for patients to receive both ChEIs and anticholinergic agents, which counteract each other. Medications with anticholinergic effects, such as diphenhydramine, tricyclic antidepressants (eg, amitriptyline, nortriptyline), and oxybutynin (commonly used for bladder spasticity), can cause cognitive dysfunction. Therefore, a careful listing of the patient's medications is important so that the physician can reduce the doses of, or ideally eliminate, all centrally acting anticholinergic agents.

Cholinesterase inhibitors (ChEIs) are used to palliate cholinergic deficiency. All 4 currently approved ChEIs (ie, tacrine, donepezil, rivastigmine, galantamine) inhibit acetylcholinesterase (AChE) at the synapse (specific cholinesterase). Tacrine was the first agent that was approved for $\mathrm{AD}$, but because of its potential to cause hepatotoxicity, it is now rarely used.

Tacrine and rivastigmine also inhibit butyrylcholinesterase (BuChE). Although BuChE levels may be increased in $\mathrm{AD}$, it is not clear that rivastigmine and tacrine have greater clinical efficacy than donepezil and galantamine.

Galantamine has a different second mechanism of action; it is also a presynaptic nicotinic modulator. No data exist to indicate that this second mechanism is of clinical importance.

\section{Donepezil (Aricept, Aricept ODT)}

Donepezil is indicated for the treatment of dementia of the Alzheimer type. Donepezil has shown efficacy in patients with mild to moderate $\mathrm{AD}$, as well as moderate to severe AD. It selectively inhibits acetylcholinesterase, the enzyme responsible for the destruction of acetylcholine, and improves the availability of acetylcholine. Donepezil's long half-life provides a long duration of drug availability for binding at the receptor sites. There is no evidence to suggest that the underlying disease process of dementia is affected by administration of donepezil.

Dosing recommendations for mild to moderate AD are 5-10 mg given once daily. Patients with moderate to severe AD can be given 10 or $23 \mathrm{mg}$ once daily.

\section{Rivastigmine (Exelon, Exelon Patch)}

Rivastigmine is indicated for the treatment of mild to moderate dementia of the Alzheimer type. Initial dosing recommendations are $1.5 \mathrm{mg}$ given twice daily, with a maximum dose of 
$12 \mathrm{mg} /$ day. Rivastigmine is a potent, selective inhibitor of brain AChE and BChE. Rivastigmine is considered a pseudo-irreversible inhibitor of AChE.

While the precise mechanism of rivastigmine's action is unknown, it is postulated to exert its therapeutic effect by enhancing cholinergic function. This is accomplished by increasing the concentration of acetylcholine through reversible inhibition of its hydrolysis by cholinesterase.

\section{Galantamine (Razadyne, Razadyne ER)}

Galantamine is indicated for the treatment of mild to moderate dementia of the Alzheimer type. It enhances central cholinergic function and likely inhibits AChE. There is no evidence that galantamine alters the course of the underlying dementing process. The dosing recommendation for the immediate-release formulation is $4 \mathrm{mg}$ twice daily. The extendedrelease formulation is given at a dose of $8 \mathrm{mg}$ once daily. The maintenance dose after dose titration is $16-24 \mathrm{mg} /$ day.

\section{Tacrine}

Tacrine was the first agent approved for AD. It is indicated in patients with mild to moderate dementia. It is associated with hepatotoxicity and is no longer commonly used. Tacrine inhibits AChE, the enzyme responsible for the destruction of acetylcholine, and improves the availability of acetylcholine. Tacrine inhibits both AChE and BChE; however, it is more selective for AChE.

\section{N-Methyl-D-Aspartate Antagonists}

The only drug in the $N$-methyl-D-aspartate (NMDA) antagonist class that is approved by the US Food and Drug Administration is memantine. This agent may be used alone or in combination with AChE inhibitors.

\section{Memantine (Namenda, Namenda XR)}

Namenda is approved for the treatment of moderate to severe dementia in patients with $\mathrm{AD}$. The initial dose for the immediate-release formulation is $5 \mathrm{mg}$ once daily, and it can be titrated to a maximum dose of $20 \mathrm{mg} /$ day. The initial dose for the extended-release formulation is $7 \mathrm{mg}$ once daily, and it can be titrated to a maximum dose of 28 $\mathrm{mg} /$ day. Side effects include dizziness, confusion, headache, constipation, nausea, and agitation.

\section{B. Treatment of moderate to severe disease}

The partial $N$-methyl-D-aspartate (NMDA) antagonist memantine (Namenda, Namenda $\mathrm{XR}$ ) is believed to work by improving the signal-to-noise ratio of glutamatergic transmission 
at the NMDA receptor. Blockade of NMDA receptors by memantine is thought to slow the intracellular calcium accumulation and thereby help prevent further nerve damage. This agent is approved by the FDA for treating moderate and severe AD.

Several studies have demonstrated that memantine can be safely used in combination with ChEIs. The combination of memantine with a ChEI has been shown to significantly delay institutionalization in AD patients (34). Studies suggest that the use of memantine with donepezil affects cognition in moderate to severe AD (35) but not in mild to moderate AD $(36,37)$. Dizziness, headache, and confusion are some of the most common side effects of memantine.

A variety of behavioral and pharmacologic interventions can alleviate clinical manifestations of $\mathrm{AD}$, such as anxiety, agitation, depression, and psychotic behavior. The effectiveness of such interventions ranges from modest and temporary to excellent and prolonged. No specific agent or dose of individual agents is unanimously accepted for the wide array of clinical manifestations. At present, the FDA has not approved any psychotropic agent for the treatment of AD.

\section{Antidepressants and mood modulators}

- Antidepressants, such as citalopram (Celexa), fluoxetine (Prozac), paroxetine (Paxil), and sertraline (Zoloft) treat irritability and mood.

- Anxiolytics, such as lorazepam (Ativan) and oxazepam (Serax) treat anxiety and restlessness.

- Antipsychotic medications, such as aripiprazole (abilify), clozapine (Clozaril), haloperidol (Haldol), and olanzapine (Zyprexa) treat hallucinations, delusions, agitation, and aggression.

Antidepressants have an important role in the treatment of mood disorders in patients with AD. Depression is observed in more than $30 \%$ of patients with $\mathrm{AD}$, and it frequently begins before AD is clinically diagnosed. Therefore, palliation of this frequent comorbid condition may improve cognitive and noncognitive performance.

Nyth found citalopram to be beneficial in mood and other neuropsychiatric symptoms in patients in the moderate stage of AD (38). Because citalopram can cause dose-dependent increases in the QT interval, the FDA recommends using a maximum of $40 \mathrm{mg}$ a day and considering $20 \mathrm{mg}$ a day in the elderly (39).

Weintraub et al (40) and Petracca et al (41) found sertraline and fluoxetine to have no shortor long-term benefit in mood over placebo. Similarly, Banerjee et al found that treatment of depression with sertraline or mirtazapine provided no benefit compared with placebo and increased the risk of adverse events (42).

Other mood modulators, such as valproic acid, can be helpful for the treatment of disruptive behaviors and outbursts of anger, which patients with moderately advanced or advanced stages of AD may have. 
Results of several studies indicate that anticonvulsants (eg, gabapentin, valproic acid) may have a role in the treatment of behavioral problems in patients with Alzheimer disease. However, a trial of 313 patients with moderate AD found that 24 months of treatment with valproate did not delay emergence of agitation or psychosis, did not slow cognitive or functional decline, and was associated A variety of experimental therapies have been proposed for AD. These include antiamyloid therapy, reversal of excess tau phosphorylation, estrogen therapy, vitamin E therapy, and free-radical scavenger therapy. Studies of these therapies have yielded mostly disappointing results.

In the past 10 years, numerous antiamyloid therapy studies have been conducted to decrease toxic amyloid fragments in the brain, including studies of the following:

- Vaccination with amyloid species

- Administration of monoclonal antiamyloid antibodies

- Administration of intravenous immune globulin that may contain amyloid-binding antibodies

- $\quad$ Selective amyloid-lowering agents

- Chelating agents to prevent amyloid polymerization

- Brain shunting to improve removal of amyloid

- Beta-secretase inhibitors to prevent generation of the A-beta amyloid fragment

To date, no phase III study of antiamyloid therapies has shown a combination of acceptable efficacy and side effects.

Growing awareness that tau is a central player in AD pathogenesis has suggested that this protein may offer an avenue for therapeutic intervention. Studies are ongoing with agents that may prevent or reverse excess tau phosphorylation and thereby diminish formation of neurofibrillary tangles (43).

Free-radical scavenger therapy has also attracted attention, because excess levels of free radicals in the brain are neurotoxic. Nonetheless, no study has demonstrated efficacy of freeradical scavengers in the treatment of the cognitive symptoms of AD.

Various studies indicate that oxidative stress may be a part of the pathogenesis of AD. In the $\mathrm{AD}$, high-dose vitamin E (2000 units per day of alpha-tocopherol) for 2 years slowed the progression of disease in patients with moderate AD (44). This benefit presumably resulted from the antioxidant effects of vitamin $\mathrm{E}$.

Subsequent studies, however, have suggested that vitamin E supplementation may increase risk of adverse cardiovascular outcomes. Therefore, use of vitamin E is not currently recommended.

Transcranial magnetic stimulation (TMS) has been used to identify therapeutic targets in AD and to monitor the effects of pharmacologic agents, and both TMS and transcranial direct current stimulation are being explored for a possible therapeutic role in AD. However, evidence of therapeutic benefit from these modalities is highly preliminary (45). 


\section{Herbal medicine is the bridge between complementary therapies \& conventional treatment}

In all cultures, the origins of herbal medicine are lost in the mists of time. There is little doubt that humans used herbs for healing well before anything could be written about them. At some point in an advancing culture, written documents become the repository for knowledge that had been passed on from one generation to the next. Among the earliest such documents are those describing the religious beliefs of the people and those describing the medical practices. Medical foods are dietary supplements intended to compensate specific nutritional problems caused by a disease or condition.

Effective pharmacological drugs for treating AD are still to be discovered. Current western pharmacological approaches against neurodegeneration in dementia develop symptomrelieving and disease-modifying drugs. Current integrative and holistic approaches of Chinese medicine to discovering drugs for neurodegeneration in dementia include (1) single molecules from the herbs, (2) standardized extracts from a single herb, and (3) herbal formula with definite composition. At present, acetylcholinesterase inhibitors (AChEI) are the first group of drugs approved by the FDA to treat mild to moderate Alzheimer's disease. Most of these drugs such as huperzine and galanthamine are originally isolated from plants. However, AChE inhibitors have limited success as they only improve memory in mild dementia but cannot stop the process of neurodegeneration; while memantine possesses neuroprotective effects only with a little ability in memory enhancement. There has been a major rush among neuroscience research institutions and pharmaceutical firms worldwide to search for safer and more effective therapeutic agents for AD.

However, mounting evidence obtained in vitro and in vivo, suggests that various traditionally used plants significantly affect key metabolic alterations culminating in ADtypical neurodegeneration.

Beside synthetic drugs, a variety of AD related medicine originates from traditionally used plants. In this respect, Ginkgo biloba and galantamine represent the most famous cases.

Indeed, the majority of recent reports on plants with traditional uses and activities relevant for $\mathrm{AD}$ originate from the traditional Chinese and Oriental Medicine, as well as from Kampo Ayurveda and Mediterranean traditional knowledge.

\section{Ginkgo Biloba - From traditional Chinese medicine to a standardized drug}

Originally, Ginkgo biloba (Coniferae) has been traditionally used for respiratory disorders in China and to improve memory loss associated with blood circulation abnormalities in Iran. This herb has been subjected to numerous investigations regarding its potential in cognitive disorders. Standardized extracts, particularly EGb 761, derived from the plants' leaves are successfully used as herbal drug for the improvement of cognitive and memory impairment. EGb 761 represents a prototype of plant extracts for attenuating Central Nervous System 
disorders, due to the fact that both flavonoids and terpenic lactones, which are partly also present in numerous other plant extracts, have been identified as the active principles in Ginkgo extracts as well as the ample experimental evidence on EGb 761's protective efficiency in vitro and in vivo. The potential of EGb 761 to attenuate the cytotoxic effects of Alzheimer's related neurotoxic amyloid peptides when added to the culture medium was demonstrated not only in neuronal-like cell lines but also primary neurons, though with different efficiency. The impact of Ginkgo extract has been largely attributed to its antioxidant activity. The effects of oxidative stress were reduced in lymphocytes and brain cells derived of EGb 761-treated AD-transgenic and non-transgenic mice. Recent data, however, indicate that Ginkgo biloba extract-761 (Gbe-761) also affects the production of neurotoxic beta-amyloid peptides $(\mathrm{A} \beta)$, for example, by up-regulating $\alpha$-secretase activity both in cells and animals.

We speculate that metabolic alterations, mediated by vasodilatory and tropic effects of EGb 761 , might be responsible for this finding.

Ginkgo biloba extract (Gbe) and two ingredients, bilobalide and ginkgolide B, are presented to the CSWG as part of a review of botanicals being used as dietary supplements in the United States. ( 1 of 3 adults in the United States is now taking dietary supplements). Sweeping deregulation of botanicals now permits GBE to be sold as a dietary supplement to a willing public eager to "improve brain functioning" or "promote radical scavenging activity.".

Gbe is a well defined product, and it or its active ingredients, the ginkgolides, especially ginkgolide B, and bilobalide, has clearly demonstrated biological activity. It can be consumed in rather large doses for an extended period of time. Under the Dietary Supplement Health and Education Act of 1994, Gbe can be sold legally if it is not labeled or accompanied by any therapeutic or health claims. Herbal remedies can be labeled with descriptions of their role in affecting physiological structure or function, but must be labeled with a disclaimer that the product has not been evaluated by the FDA for cure, prevention, or treatment of a disease.

\section{Standardized ingredients of Gbe}

The extract utilized in medicine is standardized in a multi-step procedure designed to concentrate the desired active principles from the plant. These extracts contain approximately $24 \%$ flavone glycosides (primarily composed of quercetin, kaempferol, and isorhamnetin) and $6 \%$ terpene lactones (2.8-3.4\% ginkgolides $\mathrm{A}, \mathrm{B}$, and $\mathrm{C}$, and $2.6-3.2 \%$ bilobalide). Ginkgolide B accounts for A $\beta$ out $0.8 \%$ of the total extract and bilobalide accounts for A $\beta$ out $3 \%$ of the extract. Other constituents include proanthocyanadins, glucose, rhamnose, organic acids, D-glucaric acid and ginkgolic acid (at most $5 \mathrm{ppm}$ ginkgolic acids). Much of the curative properties of Gbe are due to the activities of these flavonoids.

Human Exposure: There is potential for ingestion of Gbe to a widespread consumer population, since this product is readily available without prescription at a cost highly 
competitive with prescription medications. The recommended dose of Gbe is 120 to $160 \mathrm{mg}$ daily for persons with intermittent claudication and $240 \mathrm{mg}$ daily for cerebrovascular insufficiency, early stage Alzheimer's disease, resistant depression, and impotence.

\section{Galantamine}

Galantamine is an alkaloid known form several members of the Amaryllis family (Amaryllidaceae), and the idea for developing a medical product for AD from these species seems to be based on the local use of one of these species in a remote part of Europe. It has become an important therapeutic options used to slow down the process of neurological degeneration in $\mathrm{AD}$. Its development from little known observational studies in the Caucasus Mountains (Southern Russia), to the use of this drug in Eastern European countries (esp. Bulgaria) in the treatment of poliomyelitis and ultimately to the recent introduction onto Western markets in the treatment of AD. Galantamine was first isolated from snowdrop (Galanthus spp.) but today it is obtained from Narcissus spp. and Leucojum spp. as well as synthetically. According to unconfirmed reports, in the 1950s, a Bulgarian pharmacologist noticed the use of the common snowdrop growing in the wild by people who were rubbing it on their foreheads to ease nerve pain. Also, some of the earlier publications indicate the extensive use of snowdrop in Eastern Europe, such as Romania, Ukraine, the Balkan Peninsula, and in the Eastern Mediterranean countries. However, Mashkovsky and Kruglikova-Lvov published the first work that establishes the acetylcholine esterase inhibiting properties of galantamine isolated from Galanthus woronowii. Poliomyelitis was one of the first indications for galantamine, especially in the Eastern and Central European, since the compound enhances nerve impulse transmission at the synapse. Studies indicating blood-brain barrier penetration of the alkaloid pioneer the development of CNS-related indications. Based on the knowledge of galantamine in both the peripheral and central nervous system, many countries in Eastern Europe used it as an acknowledged treatment in Myasthenia gravis and muscular dystrophy, residual poliomyelitis paralysis symptoms, trigeminal neurologica, and other forms of neuritis. A crucial step for the success of galantamine as a medicine against $\mathrm{AD}$ was based on the synthesis developed in the mid-1990s. The scientific rationale for using cholinesterase inhibitors in the management of $\mathrm{AD}$ is based on the cholinergic hypothesis. Impairment of the central cholinergic system represents one hallmark of AD, which is characterized by loss of cholinergic neurons in the forebrain and a marked decrease in the activity of choline acetyltransferase. Overall, galantamine represents an example for the successful ethnobotany-driven development of a natural product into a clinically important drug.

In the last years, focus on AD drug discovery is shifting away from AChE inhibitors and a large number of other targets are currently being explored.

Ginseng products are popularly referred to as "adaptogen," which connotes that these products purportedly increase to physical, chemical, and biological stress and builds up general vitality, including physical and mental capacity for work. Panax ginseng roots are traditionally taken orally as adaptogens, aphrodisiacs, nourishing stimulants, and in the 
treatment of sexual dysfunction in men. The fresh root, can be directly chewed, or soaked in various wines for a period of time before drinking or chewing. Ginseng is most often availble either in whole or sliced dried form. However, usually ginseng is used at subclinical doses for a short period and as such; it does not produce measurable medicinal effects. Panax notoginseng is widely used in traditional Chinese medicine (TCM) to improve learning and memory. Moreover, protective actions against cerebral ischemia, beneficial effects on the cardiovascular system, and haemostatic, antioxidant, hypolipidemic, hepatoprotective, renoprotective, and estrogen-like activities.

\section{Galanthus woronowii (snowdrop-Galantamine)}

The delicate white flower and the Bulb of Galanthus woronowii
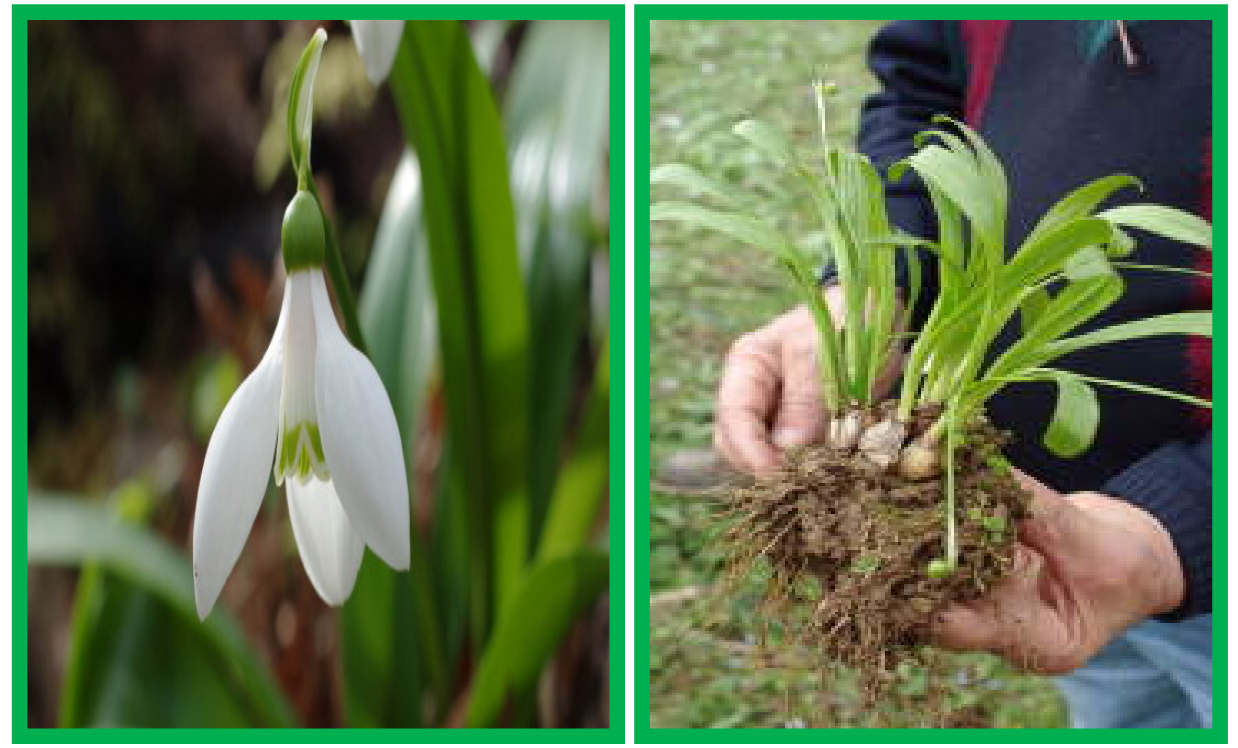

Figure 3. About this species

A snowdrop native to Turkey, Russia and Georgia, Galanthus woronowii was named in honour of the Russian botanist and plant collector Georg Woronow (1874-1931). It is popular in cultivation in Europe, and valued for its wide, green, shiny leaves, whicht provide good ground-cover and contrast with the leaves of the commonly grown snowdrop G. nivalis. Galanthus woronowii occurs from northeastern Turkey to the western and central Caucasus (Georgia and Russia). It is primarily found around the eastern Black Sea coast in the ancient provinces of Colchis and Lazistan (the Euxine Province). It occurs at 70-1,400 metres above sea level, in stony and rocky spots (on calcareous rocks, in gorges, on stony slopes and on scree), on river banks, in scrub and at forest margins, and sometimes as an epiphyte or on fallen tree trunks, rooting in moss (46-48). 


\section{Cannabinoid therapy}

The scientific literature indicates that cannabinoid therapy may provide symptomatic relief to patients afflicted with $\mathrm{AD}$ while also moderating the progression of the disease.

The intracerebroventricular administration of the synthetic cannabinoid prevented cognitive impairment and decreased neurotoxicity in rats injected with amyloid-beta peptide (49).

Additional synthetic cannabinoids were also found to reduce the inflammation associated with AD in human brain tissue in culture. In the results of Ramirez et al. 2005 indicate that cannabinoids succeed in preventing the neurodegenerative process occurring in the AD,"(50). Follow up studies by investigators demonstrated that the administration of the nonpsychotropic plant cannabinoid cannabidiol (CBD) also mitigated memory loss in a mouse model of the disease (51). Investigators at the Scripps Research Institute in California in 2006 reported that THC (Tetrahydrocannabinol (THC) is the active chemical in cannabis and is one of the oldest hallucinogenic drugs known) inhibits the enzyme responsible for the aggregation of amyloid plaque - the primary marker for AD - in a manner "considerably superior" to approved Alzheimer's drugs such as donepezil and tacrine. The investigators's results provide a mechanism whereby the THC molecule can directly impact AD pathology," researchers concluded. "THC and its analogues may provide an improved therapeutic option for $\mathrm{AD}$ by simultaneously treating both the symptoms and the progression of the disease (52).

More recently, investigators at Ohio State University, reported that older rats administered daily doses of synthetic cannabinoid [WIN 55, 212-2 $\left(\mathrm{C}_{27} \mathrm{H}_{26} \mathrm{~N}_{2} \mathrm{O}_{3} \cdot \mathrm{CH}_{3} \mathrm{SO} \mathrm{O}_{3} \mathrm{H}\right)$ ] for a period of three weeks performed significantly better than non-treated controls on a water-maze memory test. Marchalant et al. 2007, reported that rats treated with the compound experienced a 50 percent improvement in memory and a 40 to 50 percent reduction in inflammation compared to controls (53).

Previous preclinical studies have demonstrated that cannabinoids can prevent cell death by anti-oxidation. Some experts believe that cannabinoids' neuroprotective properties could also play a role in moderating AD (54). Campbell and Gowran. 2007, reported that "Cannabinoids offer a multi-faceted approach for the treatment of AD by providing neuroprotection and reducing neuroinflammation, whilst simultaneously supporting the brain's intrinsic repair mechanisms by augmenting neurotrophin expression and enhancing neurogenesis. Manipulation of the cannabinoid pathway offers a pharmacological approach for the treatment of AD that may be efficacious than current treatment regimens (55).

In addition to potentially modifying the progression of $\mathrm{AD}$, clinical trials also indicate that cannabinoid therapy can reduce agitation and stimulate weight gain in patients with the disease. Most recently, investigators at Berlin University (2006), reported that the daily administration of $2.5 \mathrm{mg}$ of synthetic THC over a two-week period reduced nocturnal motor activity and agitation in AD patients in an open-label pilot study (56). 
Clinical data presented at the 2003 annual meeting of the International Psychogeriatric Association previously reported that the oral administration of up to $10 \mathrm{mg}$ of synthetic THC reduced agitation and stimulated weight gain in late-stage Alzheimer's patients in an open-label clinical trial (57). Improved weight gain and a decrease in negative feelings among AD patients administered cannabinoids were previously reported by Volicer et al. $1997(58)$.
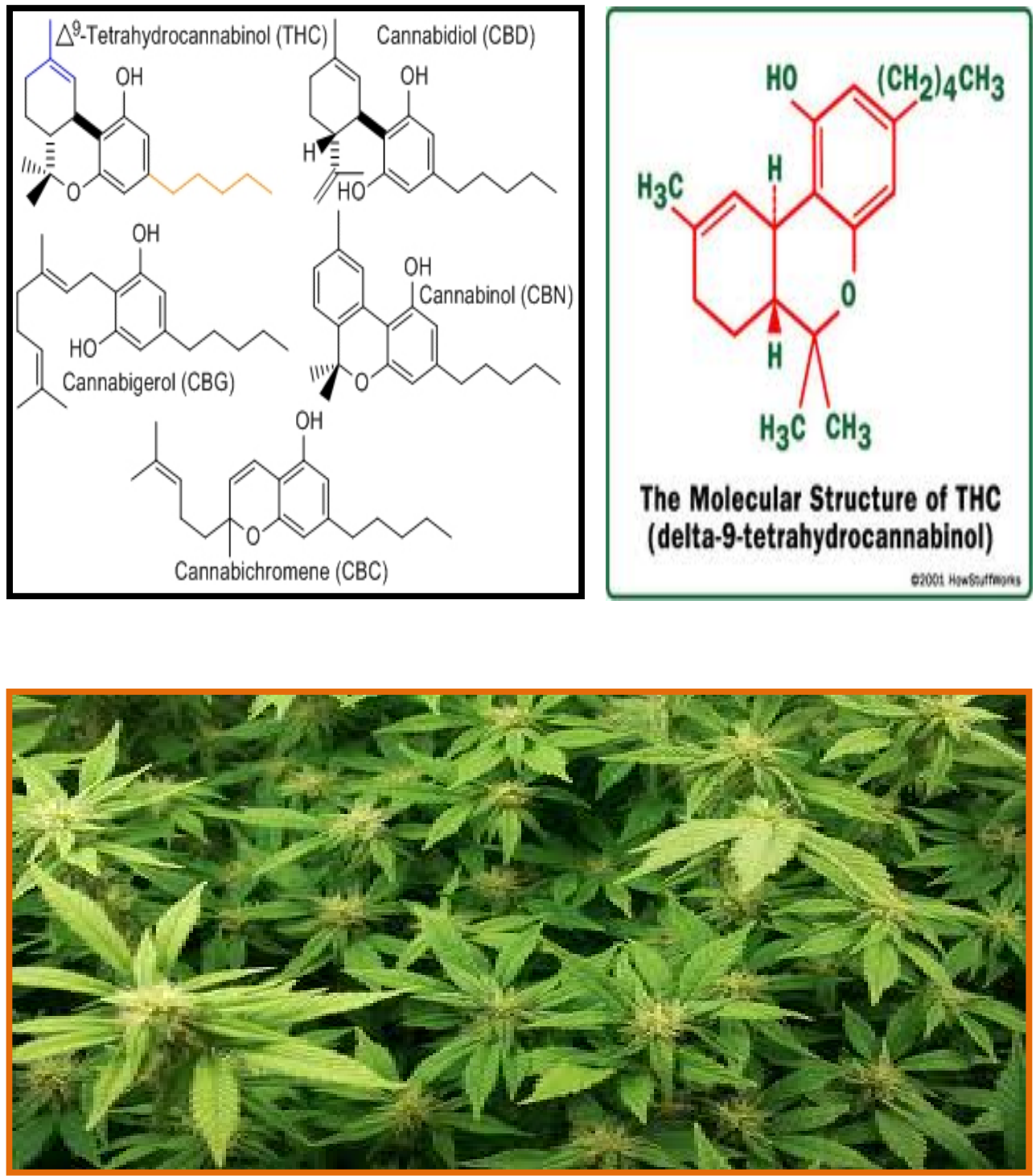


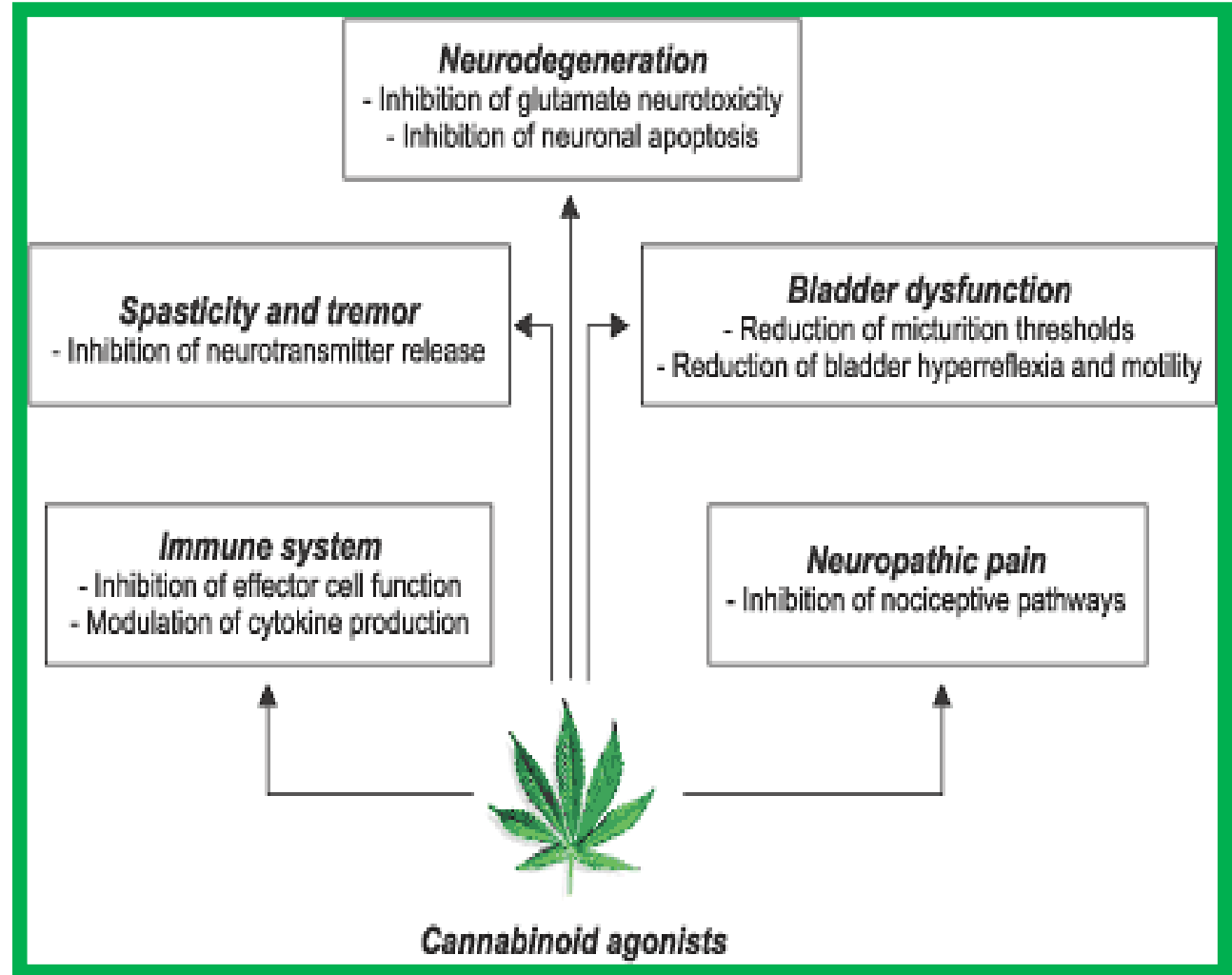

\section{Curcumin (diferuloylmethane)}

Curcumin (diferuloylmethane), a polyphenol compound responsible for the bright yellow color of turmeric, is believed to be the principal pharmacological agent. It is prepared from the roots of Curcuma longa (59). In addition to curcumin, turmeric contains the curcuminoids atlantone, bisdemethoxycurcumin, demethoxycurcumin, diaryl heptanoids, and tumerone. Turmeric also contains sesquiterpenoids and the constituent ar-tumerone (60). Other constituents include sugars, resins, proteins, vitamins, and minerals (including iron and potassium).

Due to various effects of curcumin, such as decreased Beta-amyloid plaques, delayed degradation of neurons, metal-chelation, anti-inflammatory, antioxidant and decreased microglia formation, the overall memory in patients with AD has improved (61).

Researchers found that curcumin may help the macrophages to clear the amyloid plaques found in Alzheimer's disease. Macrophages play an important role in the immune system. They help the body to fight against foreign proteins and then effectively clear them. Curcumin was treated with macrophages in blood taken from nine volunteers: six AD patients and three healthy controls. Beta amyloid was then introduced. The AD patients, 
whose macrophages were treated with curcumin, when compared with patients whose macrophages were not treated with curcumin, showed an improved uptake and ingestion of the plaques. Thus, curcumin may support the immune system to clear the amyloid protein $(61,62)$.
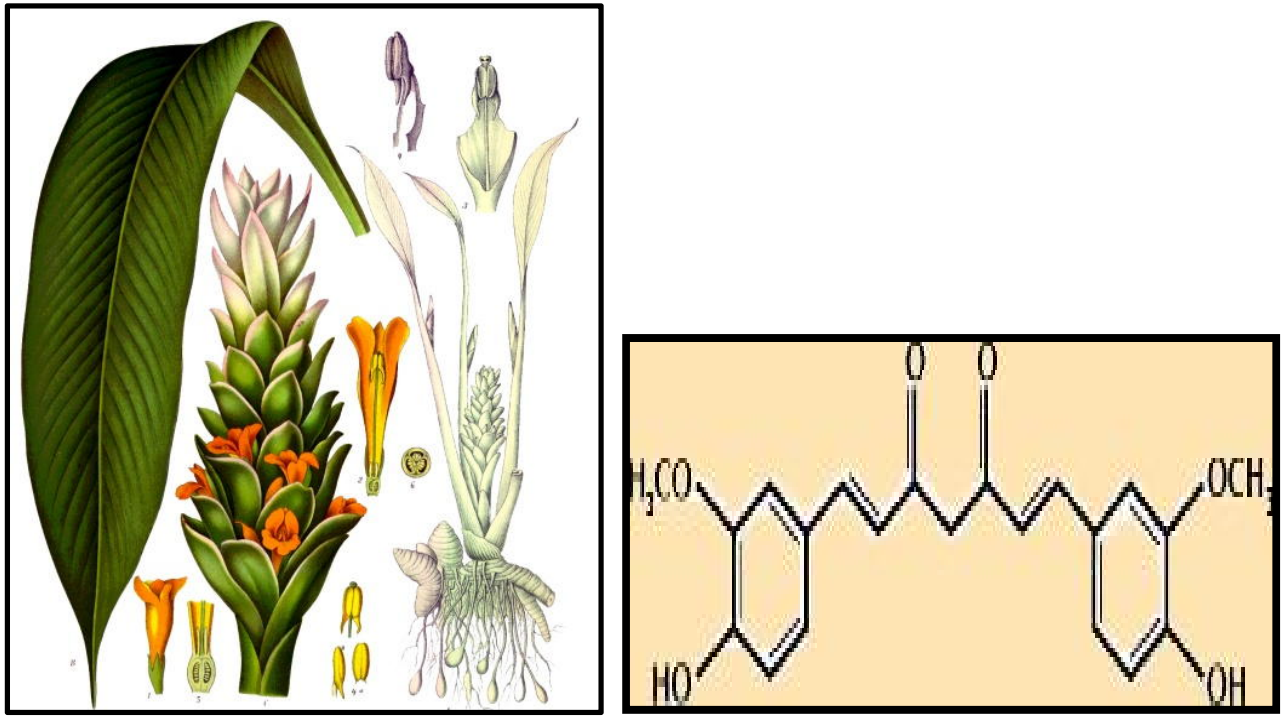

Figure 4. Curcumin (diferuloylmethane).

In addition, Curcumin has powerful antioxidant and anti-inflammatory properties; according to the scientists, these properties believe help ease Alzheimer's symptoms caused by oxidation and inflammation (63). It is well known that $A \beta$ induced oxidative stress which is a well-established pathway of neuronal cell death in AD (64). Three curcuminoids from turmeric (Curcuma longa L.), including curcumin, demethoxycurcumin, and bisdemethoxycurcumin, were found to protect PC12 rat pheochromocytoma and normal human umbilical vein endothelial (HUVEC) cells from beta $A(1-42)$ insult. These compounds may protect the cells from beta $A(1-42)$ insult through antioxidant pathways. Other animal studies of $\mathrm{AD}$ also suggest that curcumin may reduce levels of amyloid and oxidized proteins and prevent cognitive deficits (65). One alternative mechanism of action for these effects suggested by Baum et al (65), is metal chelation, which may reduce amyloid aggregation or oxidative neurotoxicity. Since curcumin more readily binds the redox-active metals and than the redox-inactive, curcumin might exert a net protective effect against beta toxicity or might suppress inflammatory damage by preventing metal induction of NFkappaB. Mouse studies that evaluated the effects of dietary curcumin on inflammation, oxidative damage, and plaque pathology demonstrated that both low and high doses of curcumin significantly lowered oxidized proteins and interleukin-1beta, which is a proinflammatory cytokine elevated in the brains of these mice (66). Low-dose but not highdose curcumin treatment has been shown to reduce the astrocytic marker GFAP and 
significantly decrease insoluble $(A \beta)$, soluble Abeta, and plaque burden by $43-50 \%$. However, levels of amyloid precursor (APP) in the membrane fraction were not reduced.

\section{Huperzine A (Qian Ceng Ta)}

Huperzine A, shows promise for enhancing memory and protecting cognitive functions and may improve cognition in AD.

Huperzine- $A$ is a new supplement derived from an ancient traditional Chinese herbal medicine that offers hope to those suffering from $\mathrm{AD}$ and other age-related mental conditions (67).

Existing evidence suggests that patients with $\mathrm{AD}$ who have taken Huperzine $A$ have improved general cognitive function, global clinical status, functional performance and reduced behavioural disturbance compared to patients taking placebos. In addition to benefiting patients suffering from Alzheimer's, Huperzine's memory-enhancing properties suggest that it may be an effective agent for improving memory and learning in healthy humans as well. Huperzine A is a natural compound derived from an ancient Chinese remedy, Qian Ceng Ta. This traditional herbal medicine was prepared from Huperzia serrata, a clubmoss that grows on the ground in damp forests and rock crevices. Brewed as an herbal tea, Qian Ceng Ta has been used in China to treat fever, inflammation, and irregular menstruation, and has been used as a diuretic.

In the late 1980's, researchers in China discovered that a purified alkaloid extracted from Huperzia, Huperzine A, was a potent, reversible inhibitor of acetylcholinesterase (AChE). Huperzine A readily crosses the blood-brain barrier to prevent acetylcholinesterase (AchE) from destroying acetylcholine. Indeed, part of the damage involved in AD is a loss of acetylcholine-containing neurons in the basal forebrain. This suggests that drugs that could inhibit cholinesterase, which breaks down acetylcholine, could increase the ability of remaining cholinergic neurons. Scientists know that Huperzine A can block acetyl cholinesterase and that it can work both in the peripheral and central nervous systems (68).

Scientists had previously learned that AchE inhibitors such as tacrine and donepezil worked by sliding into the AChE molecule to "jam up" its molecular machinery and impair its ability to degrade acetylcholine $(69,70)$.

In summary, Huperzine A is rapidly absorbed when taken orally, and possesses a very slow rate of dissociation from the enzyme and a longer duration of action. Studies in rodents show that AChE remains inhibited by 33\% after 6 hours. Huperzine A has a strong specificity for AChE, and is exceptionally well-suited to its new role, fitting into the active sites of acetylcholinesterase much like a key slipping into a lock. "Hup-A appears to bind more tightly and specifically to acetylcholinesterase than the other AChE inhibitors". This makes it a promising agent for treating various forms of dementia including AD (71-73). 

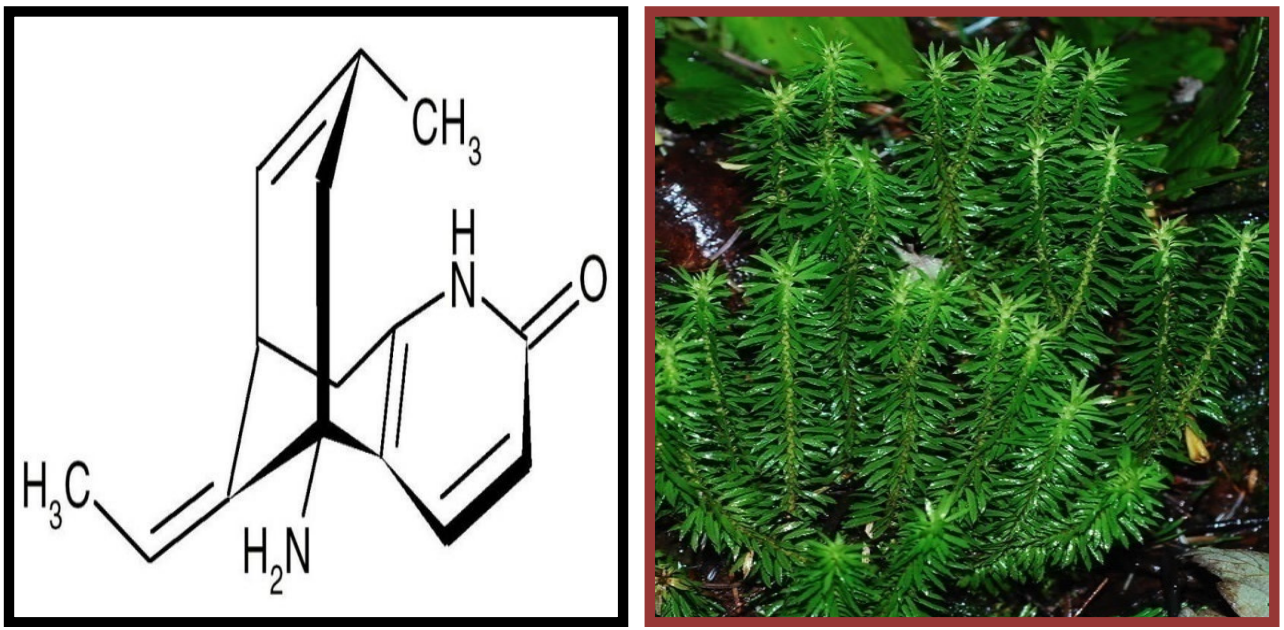

\section{Rosemary (Rosmarinus officinalis) - used as Aromatherapy}

Aromatherapy uses essential oils from plants, either applied in a lotion and absorbed by the skin or inhaled and absorbed into the lungs and nasal passages, to improve physical and mental health. Aromatic oils from plants have been used for over 5,000 years: To protect against stroke and other neurodegenerative diseases such as Alzheimer's and Lou Gehrig's disease. Rosemary contains carnosic acid, a powerful antioxidant, which helps to fight off free radicals in the brain. Carnosic acid stimulates the synthesis of Nerve Growth Factor (NGF) which may help prevent nerve cell deterioration in Alzheimer's (74).Rosmarinus officinalis is a woody evergreen native to the Mediterranean and a universal symbol of remembrance used to honor those who have passed on. The tradition of laying sprigs of rosemary across the coffin or upon a tombstone dates back to ancient Egypt. This custom continued well into the medieval period and beyond. For instance, Shakespeare's Juliet was bestowed with rosemary upon her untimely death. In Australia, where Anzac Day is celebrated in remembrance of one's family ancestors, it is still customary to wear sprigs of rosemary today. Rosemary is also associated with enhancing memory and recall. Shakespeare's Ophelia petitions Hamlet with, "There's rosemary, that's for remembrance, pray you love, remember." Scholars of ancient Greece wore wreaths of rosemary about the brow to help improve recall while taking exams. This reputation has earned the herb a place among traditional wedding herbs used to grace the bride's bouquet, headpiece, and dress. Wedding guests are also given sprigs of rosemary to wear to help them remember the occasion. It was also once common to add rosemary to the couple's wine to help them remember their sacred vows to each other. At one time, it was customary for the bride and groom to plant rosemary near the marital threshold on their day of matrimony. However, the old saying "where rosemary flourished, the woman ruled," prompted some husbands to pluck the plant from the ground lest anyone should think he wasn't fit to rule the roost. Perhaps this is why the practice fell out of favor by the late 15th century. Rosemary takes its name from the 
Latin ros maris, which means "dew of the sea." This is likely in reference to the herb's preference for growing along the seashore of its indigenous domain. The Spanish began to call the plant Romero because they believed that another Mediterranean native took refuge beneath a large rosemary bush to shelter herself and her young son as they fled to Egypt to escape Herod. In honor of this brave, young woman, the plant came to be known as Rose of Mary, which was eventually shortened to the modern name familiar to us today.

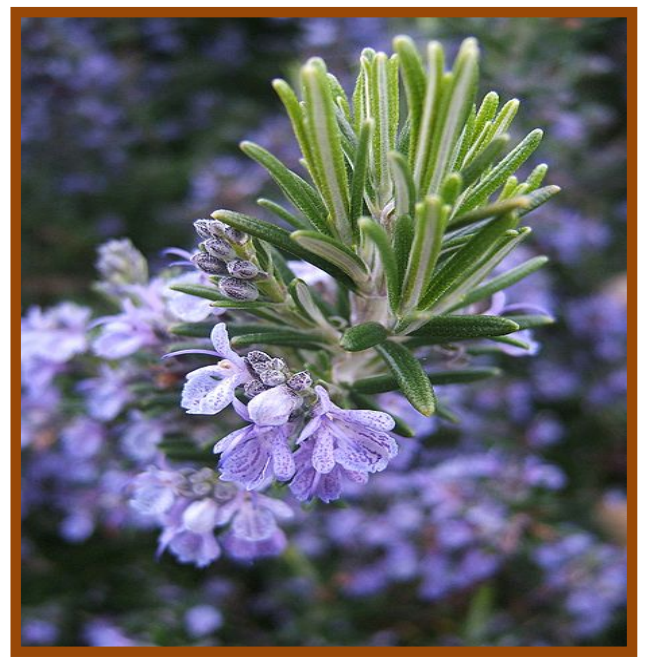

During the Middle Ages, rosemary was thought to be capable of dispelling negativity. As such, it was tucked under pillows to thwart nightmares and visits from evil spirits. It was also burned in the house to keep the black plague from entering. Perhaps this association with protection is why rosemary is still a common ingredient in incense used to cleanse sacred spaces. It was also thought to promote prosperity. In fact, 16th century merchants would often hire perfumers to infuse their shops with spirits of rosemary. The herb was also a popular addition to nosegays, wreaths, and other floral displays to encourage happiness of home and hearth.

Medicinally, rosemary has a wealth of uses, both old and new. In one of the earliest herbals known to be printed in England, Rycharde Banckes recommended that one gather leaves of rosemary and "...boyle them in fayre water and drinke that water for it is much worthe against all manner of evils in the body." Indeed, rosemary was once thought to be a cure for poor digestion, migraine, joint disorders, and muscle aches. In fact, Queen Elizabeth of Hungary was reputedly cured of semi-paralysis when she sipped a concoction of rosemary to ease her painful joints. Hence, this formula came to be known as the infamous Hungary Water. Today, rosemary is recognized as possessing several medicinal properties. For one thing, the plant contains salicylic acid, the forerunner of aspirin. This may explain why massaging the oil of rosemary into joints effectively eases arthritic or rheumatic pain. It also contains antibacterial and antimicrobial agents, and is used by modern herbalists to treat a variety of skin disorders, including dandruff. Rosemary is also being studied for its 
potential anti-cancer effects since initial studies indicate that its compounds inhibit carcinogenic chemicals from binding to cellular DNA. Rosemary may also become useful in preventing and treating Alzheimer's disease in the near future. Researchers have discovered that certain phytochemicals in the herb prevent the degradation of acetylcholine, an important brain chemical needed for normal neurotransmission. A deficiency of this chemical is commonly seen in Alzheimer's patients.

The majority of recent reports on plants with traditional uses and activities relevant for AD originate from the traditional Chinese and Oriental Medicine, as well as from Kampo Ayurveda and Mediterranean traditional knowledge. They are many plants useful for the treatment of Neurodegenerative diseases and they are many athor still to be discovered.

\section{Author details}

Bowirrat Abdalla, Mustafa Yassin, Menachem Abir, Bishara Bisharat and Zaher Armaly EMMS Nazareth-The Nazareth Hospital, Nazareth

\section{References}

[1] Bowirrat A, Friedland RP, Farrer L, Baldwin C, Korczyn A. Genetic and Environmental Risk factors for Alzheimer's disease in Israeli Arabs. Journal of Molecular Neuroscience. 2002; 19(1-2):239-245.

[2] Bowirrat A, Treves TA, Friedland RP, Korczyn AD. Prevalence of Alzheimer's Type Dementia in an elderly Arab population. European Journal of Neurology 2001; 8(2):119123.

[3] Alzheimer's Association. 2010 Alzheimer's disease facts and figures. Alzheimers Dement. 2010;6(2):158-94.

[4] Maurer K, Maurer U. Alzheimer: The Life of a Physician and Career of a Disease. New York: Columbia University Press; 2003.

[5] Alzheimer A. Uber eine eigenartige Erkangkung der Hirnrinde. In: Allgemeine Zeitschrift fur Psychiatrie und Psychisch-Gerichtliche Medizin. 64. 1907:146-148.

[6] Haass C, Selkoe DJ. Soluble protein oligomers in Neurodegeneration: lessons from the Alzheimer's amyloid I [beta] I-peptide. Nature Reviews Molecular Cell Biology. 2007; 8, 101-112.

[7] Braak H, Braak E. Neuropathological stageing of Alzheimer-related changes. Acta Neuropathol. 1991;82(4):239-59.

[8] Serrano-Pozo A, Frosch MP, Masliah E, Hyman BT. Neuropathological alterations in Alzheimer disease.Cold Spring Harb Perspect Biol. 2011;3(9):a006189.

[9] Brayne C, Richardson K, Matthews FE, et al. Neuropathological correlates of dementia in over-80-year-old brain donors from the population-based Cambridge city over-75s cohort (CC75C) study. J Alzheimers Dis. 2009;18(3):645-58.

[10] Swerdlow RH, Khan SM. The Alzheimer's disease mitochondrial cascade hypothesis: an update. Exp Neurol. 2009;218(2):308-15. 
[11] Braak H, Thal DR, Ghebremedhin E, Del Tredici K. Stages of the pathologic process in Alzheimer disease: age categories from 1 to 100 years. J Neuropathol Exp Neurol. 2011;70(11):960-9.

[12] Braak H, Braak E, Grundke-Iqbal I, Iqbal K. Occurrence of neuropil threads in the senile human brain and in Alzheimer's disease: a third location of paired helical filaments outside of neurofibrillary tangles and neuritic plaques. Neurosci Lett. 24 1986;65(3):3515.

[13] Davinelli S, Intrieri M, Russo C, Di Costanzo A, Zella D, Bosco P, et al. The "Alzheimer's disease signature": potential perspectives for novel biomarkers. Immun Ageing. 2011;8:7.

[14] Higgins GC, Beart PM, Shin YS, Chen MJ, Cheung NS, Nagley P. Oxidative stress: emerging mitochondrial and cellular themes and variations in neuronal injury. $J$ Alzheimers Dis. 2010; Suppl 2:S453-73.

[15] Ding Q, Dimayuga E, Keller JN. Oxidative damage, protein synthesis, and protein degradation in Alzheimer's disease. Curr Alzheimer Res. 2007;4(1):73-9.

[16] Rocchi A, Orsucci D, Tognoni G, Ceravolo R, Siciliano G. The role of vascular factors in late-onset sporadic Alzheimer's disease. Genetic and molecular aspects. Curr Alzheimer Res. 2009;6(3):224-37.

[17] S Roriz-Filho J, Sá-Roriz TM, Rosset I, Camozzato AL, Santos AC, Chaves ML, et al. (Pre)diAßetes, brain aging, and cognition. Biochim Biophys Acta. 2009;1792(5):432-43.

[18] Naderali EK, Ratcliffe SH, Dale MC. Obesity and Alzheimer's disease: a link between body weight and cognitive function in old age. Am J Alzheimers Dis Other Demen. 2010;24(6):445-9.

[19] de la Monte SM. Insulin resistance and Alzheimer's disease. BMB Rep. 2009;42(8):47581.

[20] Perl DP. Relationship of aluminum to Alzheimer's disease. Environ Health Perspect. 1985;63:149-53.

[21] Perl DP, Moalem S. Aluminum and Alzheimer's disease, a personal perspective after 25 years. J Alzheimers Dis. 2006;9(3 Suppl):291-300.

[22] Goldbourt U, Schnaider-Beeri M, Davidson M. Socioeconomic status in relationship to death of vascular disease and late-life dementia. J Neurol Sci. 2007;257(1-2):177-81.

[23] McDowell I, Xi G, Lindsay J, Tierney M. Mapping the connections between education and dementia. J Clin Exp Neuropsychol. 2007;29(2):127-41.

[24] Szekely CA, Zandi PP. Non-steroidal anti-inflammatory drugs and Alzheimer's disease: the epidemiological evidence. CNS Neurol Disord Drug Targets. 2010;9(2):132-9.

[25] Goldman JS, Hahn SE, Catania JW, LaRusse-Eckert S, Butson MB, Rumbaugh M, et al. Genetic counseling and testing for Alzheimer disease: joint practice guidelines of the American College of Medical Genetics and the National Society of Genetic Counselors. Genet Med. 2011;13(6):597-605.

[26] Hollingworth $P$, Harold D, Sims R, et al. Common variants at ABCA7, MS4A6A/MS4A4E, EPHA1, CD33 and CD2AP are associated with Alzheimer's disease. Nat Genet. 2011;43(5):429-35. 
[27] Caselli RJ, Dueck AC. APOE varepsilon2 and presymptomatic stage Alzheimer disease: how much is not enough?. Neurology. 2010;75(22):1952-3.

[28] Chiang GC, Insel PS, Tosun D, et al. Hippocampal atrophy rates and CSF biomarkers in elderly APOE2 normal subjects. Neurology. 2010;75(22):1976-81.

[29] Finch CE, Morgan TE. Systemic inflammation, infection, ApoE alleles, and Alzheimer disease: a position paper. Curr Alzheimer Res. 2007;4(2):185-9.

[30] Kavanagh S, Gaudig M, Van Baelen B, et al. Galantamine and behavior in Alzheimer disease: analysis of four trials. Acta Neurol Scand. 2011;124(5):302-8.

[31] Farlow M, Veloso F, Moline M, et al. Safety and tolerAßility of donepezil $23 \mathrm{mg}$ in moderate to severe Alzheimer's disease. BMC Neurol. 2011;11:57.

[32] Starr JM. Cholinesterase inhibitor treatment and urinary incontinence in Alzheimer's disease. J Am Geriatr Soc. 2007;55(5):800-1.

[33] Gill SS, Anderson GM, Fischer HD, Bell CM, Li P, Normand SL, et al. Syncope and its consequences in patients with dementia receiving cholinesterase inhibitors: a population-based cohort study. Arch Intern Med. 2009;169(9):867-73.

[34] Lachaine J, Beauchemin C, Legault M, Bineau S. Economic evaluation of the impact of memantine on time to nursing home admission in the treatment of Alzheimer disease. Can J Psychiatry. 2011;56(10):596-604.

[35] Schmitt FA, van Dyck CH, Wichems $\mathrm{CH}$, Olin JT. Cognitive response to memantine in moderate to severe Alzheimer disease patients already receiving donepezil: an exploratory reanalysis. Alzheimer Dis Assoc Disord. 2006;20(4):255-62.

[36] Porsteinsson AP, Grossberg GT, Mintzer J, Olin JT. Memantine treatment in patients with mild to moderate Alzheimer's disease already receiving a cholinesterase inhibitor: a randomized, double-blind, placebo-controlled trial. Curr Alzheimer Res. 2008;5(1):83-9.

[37] Schneider LS, Dagerman KS, Higgins JP, McShane R. Lack of evidence for the efficacy of memantine in mild Alzheimer disease. Arch Neurol. 2011;68(8):991-8.

[38] Nyth AL, Gottfries CG. The clinical efficacy of citalopram in treatment of emotional disturbances in dementia disorders. A Nordic multicentre study. Br J Psychiatry. 1990;157:894-901.

[39] US Food and Drug Administration. August 24, 2011. FDA Drug Safety Communication: A $\beta$ normal heart rhythms associated with high doses of Celexa (citalopram hydrobromide). Available at http://www.fda.gov/Drugs/DrugSafety/ucm269086.htm, 2011.

[40] Weintraub D, Rosenberg PB, Drye LT, et al. Sertraline for the treatment of depression in Alzheimer disease: week-24 outcomes. Am J Geriatr Psychiatry. 2010;18(4):332-40.

[41] Petracca GM, Chemerinski E, Starkstein SE. A double-blind, placebo-controlled study of fluoxetine in depressed patients with Alzheimer's disease. Int Psychogeriatr. 2001;13(2):233-40.

[42] Banerjee S, Hellier J, Dewey M, et al. Sertraline or mirtazapine for depression in dementia (HTA-SADD): a randomised, multicentre, double-blind, placebo-controlled trial. Lancet. 2011;378(9789):403-11.

[43] Pritchard SM, Dolan PJ, Vitkus A, Johnson GV. The toxicity of tau in Alzheimer disease: turnover, targets and potential therapeutics. J Cell Mol Med. 2011;15(8):1621-35. 
[44] Sano M, Ernesto C, Thomas RG, et al. A controlled trial of selegiline, alpha-tocopherol, or both as treatment for Alzheimer's disease. The Alzheimer's Disease Cooperative Study. N Engl J Med. 1997;336(17):1216-22.

[45] Freitas C, Mondragón-Llorca H, Pascual-Leone A. Noninvasive brain stimulation in Alzheimer's disease: systematic review and perspectives for the future. Exp Gerontol. 2011;46(8):611-27.

[46] Bishop, M., Davis, A.P \& Grimshaw, J. (2006). Snowdrops: A Monograph of Cultivated Galanthus. Griffin Press, Cheltenham.

[47] Davis, A.P. (1999). The Genus Galanthus. Royal Botanic Gardens, Kew in association with Timber Press, Oregon.

[48] Heinrich, M. \& Teoh, H. L. Galanthamine from snowdrop - the development of a modern drug against Alzheimer's disease from local Caucasian knowledge. J. Ethnopharmacol. 2004; 92: 147-162.

[49] Ramirez et al. 2005. Prevention of Alzheimer's disease pathology by cannabinoids. The Journal of Neuroscience. 2005; 25: 1904-1913.

[50] Israel National News. December 16, 2010. "Israeli research shows cannabidiol may slow Alzheimer's disease."

[51] Eubanks et al. A molecular link between the active component of marijuana and Alzheimer's disease pathology. Molecular Pharmaceutics. 2006; 3: 773-777.

[52] Marchalant et al. Anti-inflammatory property of the cannabinoid agonist WIN-55212-2 in a rodent model of chronic brain inflammation. Neuroscience,2007; 144: 1516-1522.

[53] Hampson et al. 1998. Cannabidiol and delta-9-tetrahydrocannabinol are neuroprotective antioxidants.Proceedings of the National Academy of Sciences. 1998; 95: 8268-8273.

[54] Science News. 1998. "Marijuana chemical tapped to fight strokes."

[55] Campbell and Gowran. Alzheimer's disease; taking the edge off with cannabinoids? British Journal of Pharmacology. 2007; 152: 655-662.

[56] Walther et al. Delta-9-tetrahydrocannabinol for nighttime agitation in severe dementia.Physcopharmacology. 2006; 185: 524-528.

[57] BBC News. August 21, 2003. " Cannabis lifts Alzheimer's appetite."

[58] Volicer et al. Effects of dronabinol on anorexia and disturbed behavior in patients with Alzheimer's disease. International Journal of Geriatric Psychiatry. 1997; 12: 913-919.

[59] Ali, R. E. and Rattan, S. I. Curcumin's biphasic hormetic response on proteasome activity and heat-shock protein synthesis in human keratinocytes. Ann N Y Acad Sci. 2006;1067:394-399.

[60] Ji, M., Choi, J., Lee, J., and Lee, Y. Induction of apoptosis by ar-turmerone on various cell lines. Int J Mol Med. 2004;14(2):253-256.

[61] Shrikant Mishra and Kalpana Palanivelu. The effect of curcumin (turmeric) on Alzheimer's disease: An overview. Ann Indian Acad Neurol. 2008; 11(1): 13-19.

[62] Zhang L, Fiala M, Cashman J, Sayre J, Espinosa A, Mahanian M, et al. Curcuminoids enhance amyloid -beta uptake by macrophages of Alzheimer's disease patients. J Alzheimers Dis. 2006;10:1-7. 
[63] Frautschy SA, Hu W. Phenolic anti inflammatory antioxidant reversal of b induced cognitive deficits and neuropathology. Neurobiol Aging. 2001;22:993-1005.

[64] Kim, D. S., Park, S. Y., and Kim, J. K. Curcuminoids from Curcuma longa L. (Zingiberaceae) that protect PC12 rat pheochromocytoma and normal human umbilical vein endothelial cells from betaA(1-42) insult. Neurosci Lett 4-27-2001;303(1):57-61.

[65] Baum, L. and Ng, A. Curcumin interaction with copper and iron suggests one possible mechanism of action in Alzheimer's disease animal models. J Alzheimers Dis 2004;6(4):367-377.

[66] Lim, G. P., Chu, T., Yang, F., Beech, W., Frautschy, S. A., and Cole, G. M. The curry spice curcumin reduces oxidative damage and amyloid pathology in an Alzheimer transgenic mouse. J Neurosci. 2001;21(21):8370-8377.

[67] Xu SS; Gao ZX; Weng Z; Du ZM; Xu WA; Yang JS; Zhang ML; Tong ZH; Fang YS;Chai $\mathrm{XS}$; et al, Efficacy of tablet huperzine-A on memory' cognition' and behavior in Alzheimer`s disease. Chung Kuo Yao Li, Hsueh Pao16:391-5, 1995.

[68] Raves ML, Harel M, Pang YP, Silman I, Kozikowski AP, Sussman JL. Structure of acetylcholinesterase complexed with the nootropic alkaloid, (-)-huperzine A. Nat Struct Biol. 1997;4(1):57-63.

[69] Watkins PB, Zimmerman HJ, Knapp MJ. Hepatotoxic effects of tacrine administration in patients with Alzheimer's disease. JAMA. 1994; 271:992-8.

[70] Raves ML, Harel M, Pang YP, Silman I, Kozikowski AP, Sussman JL. Structure of acetylcholinesterase complexed with the nootropic alkaloid, (-)-huperzine A. Nat Struct Biol. 1997;4(1):57-63.

[71] Qian BC, Wang M, Zhou ZF, Chen K, Zhou RR, Chen GS. Pharmacokinetics of tablet huperzine A in six volunteers. Chung Kuo Yao Li Hsueh Pao. 1995;16(5):396-8.

[72] Mazurek, A. An open 1A $\beta$ el trial of Huperzine A in the treatment of Alzheimer's disease. Alternative Therapies. 1999; 5(2):97.

[73] Saxena A, Qian N, Kovach IM, Kozikowski AP, Pang YP, Vellom DC, Radic Z, Quinn D, Taylor P, Doctor BP. Identification of amino acid residues involved in the binding of Huperzine A to cholinesterases. Protein Sci. 1994;3(10):1770-8.

[74] Jimbo D, Kimura Y, Taniguchi M, Inoue M, Urakami K.Effect of aromatherapy on patients with Alzheimer's disease. Psychogeriatrics. 2009; 9(4):173-9. 